REVISTA BRASILEIRA DE ANÁLISE DO COMPORTAMENTO / BRAZILIAN JOURNAL OF BEHAVIOR ANALYSIS, 2014, Vol. 10, №.1, $33-38$.

\title{
INTERFERÊNCIA DE CONTROLE PELA POSIÇÃO DO ESTÍMULO EM TREINO DE DISCRIMINAÇÕES CONDICIONAIS ARBITRÁRIAS EM MACACO-PREGO (Sapajus sp.)
}

\section{INTERFERENCE OF CONTROL BY STIMULUS POSITION IN ARBITRARY CONDITIONAL DISCRIMINATION TRAINING IN CAPUCHIN MONKEY (Sapajus sp.)}

\author{
KATARINA KATAOKA DIAS \\ ANA LEDA DE FARIA BRINO \\ ROMARIZ DA SILVA BARROS \\ OLAVO DE FARIA GaLVÃO \\ UNIVERSIDADE FEDERAL DO PARÁ, BRASIL E \\ INSTITUTO NACIONAL DE CIÊNCIA E TECNOLOGIA SOBRE COMPORTAMENTO COGNIÇÃo E ENSINO, BRASIL
}

\begin{abstract}
RESUMO
A interferência do controle pela posição dos estímulos no treino de discriminações condicionais já foi documentada na literatura sobre controle de estímulos com animais. Estudos subsequentes sugeriram (1) a introdução da variação da posição do estímulo modelo e (2) a ampliação da variação da posição dos estímulos de comparação. O presente estudo documenta a interferência do controle pela posição dos estímulos mesmo nessas condições de grande variação da posição dos estímulos no treino. Foi utilizado o procedimento de matching-to-sample arbitrário com atraso zero com um macaco-prego (Sapajus sp.). A posição dos estímulos modelo e de comparação, apresentados na tela sensível ao toque de um computador, variava a cada tentativa, podendo assumir qualquer uma das nove posições de uma matriz $3 \times 3$. Durante o treino, foram observados erros sistemáticos nas configurações em tentativas em que a comparação correta ( $\mathrm{S}+$ ) se localizava nas posições 8 ou 9. Manipulações nas posições nas quais o S+ era apresentado confirmaram a interferência do controle pela posição. É provável que esse tipo de interferência esteja envolvido em boa parte dos fracassos obtidos em estudos visando demonstrar identidade generalizada e a formação de classes de equivalência em não-humanos.

Palavras-chave: relações de controle de estímulos, discriminações condicionais arbitrárias, Sapajus sp.
\end{abstract}

The interference of the control by stimulus locations in conditional discrimination training has already been documented in the literature on stimulus control with animals. Subsequent studies suggested (1) variation of the position in which sample stimuli are presented and (2) increasing variation of comparison stimulus positions. The present study documents the interference of the control by stimulus position even in such condition of great variation of stimulus locations. Arbitrary zero-delay matching-to-sample was carried out with a capuchin monkey (Sapajus sp.). The position of sample and comparison stimuli, presented on the computer screen varied every trial among nine positions in a $3 \times 3$. During training, we observed systematic errors in trials in which correct comparison (S+) was located at Positions 8 and 9. Manipulations in the positions in which the stimuli were presented confirmed the interference of control by the position. It is possible that such kind of interference causes part of the difficulties in studies with the purpose of demonstrating generalized identity matching and equivalence class formation in non-human subjects.

Keywords: stimulus control relations, arbitrary conditional discriminations, Sapajus sp.

O presente trabalho deriva de pesquisa financiada pelo CNPq (Processo \#573972/2008-7) e pela FAPESP (Processo \#2008/57705-8) no âmbito do Instituto Nacional de Ciência e Tecnologia sobre Comportamento, Cognição e Ensino (INCT-ECCE). Os autores agradecem Klena Sarges pela atenção à manutenção da saúde dos animais e Adilson Pastana pelo excelente manejo dos animais.

Correspondência para: katarinakataoka@gmail.com 
Num treino discriminativo envolvendo tentativas com um S+ e um S- apresentados simultaneamente, é possível, sob certas condições, identificar com clareza quais aspectos do estímulo discriminativo apresentado adquiriram o controle do responder sobre as respostas de escolha do S+. Em um experimento clássico de Reynolds (1961), respostas de bicar de dois pombos eram reforçadas na presença de triângulo sobre vermelho $(\mathrm{S}+)$ e não reforçadas na presença de círculo sobre verde (S-). Quando as cores e as formas dos estímulos compostos foram apresentadas separadamente, observou-se que um dos pombos respondia sob controle da cor e que o outro pombo respondia sob controle da forma.

Além das propriedades do estímulo nominal, outros aspectos do contexto de treino podem adquirir o controle sobre o responder. Considere-se, por exemplo, um treino de discriminação auditivo visual com uma criança diagnosticada com autismo. $\mathrm{O}$ educador/experimentador apresenta a figura de um cachorro à esquerda do participante e a figura de um gato à direita do participante. Então, o experimentador diz "cachorro". A criança escolhe a figura da esquerda e essa escolha é reforçada. Como resultado, a resposta reforçada pode ter ficado sob controle da posição de apresentação da figura e não de suas características nominais. Desse modo, quando as posições das figuras são invertidas, numa próxima tentativa do treino, o participante pode continuar escolhendo a figura da esquerda, qualquer que seja ela, e independentemente de qual seja estímulo auditivo apresentado pelo experimentador/educador.

A interferência do controle não programado pela posição dos estímulos já foi claramente documentada em procedimentos de treino de relações condicionais com sujeitos não-humanos (Iversen, 1997; Iversen, Sidman, \& Carrigan, 1986; Lionello \& Urcuioli, 1998; Sidman, 1992). Usualmente, um treino de emparelhamento ao modelo por identidade ocorre com o estímulo modelo sendo apresentado numa janela central e dois estímulos de comparação em duas janelas laterais. Após a aquisição das discriminações, um teste de movimentação do estímulo modelo é realizado, de forma que esse estímulo passa a ser apresentado em quaisquer das três janelas. Após a resposta de observação ao modelo, os estímulos de comparação são apresentados nas janelas remanescentes. Esses estudos encontraram que a precisão da discriminação se deteriora com a apresentação dos estímulos em novas posições, mostrando que, para os sujeitos, a posição dos estímulos nominais fazia parte do controle das respostas de escolha.

Embora nesses estudos o procedimento pudesse ser nomeado como "emparelhamento ao modelo por identidade", o desempenho treinado não podia ser descrito pelo mesmo nome. Esses estudos apontam pelo menos dois exemplos de relações de controle de estímulo incoerentes com o planejamento experimental: 1) O controle pelas posições individuais dos estímulos nas tentativas, e 2) $\mathrm{O}$ controle pelas configurações formadas por vários estímulos apresentados simultaneamente.
O controle de respostas por dimensões não planejadas dos estímulos tem sido referido sob o título de "Teoria da Coerência de Topografias de Controle de Estímulos" (Dube \& McIlvane, 1996; McIlvane \& Dube, 2003; McIlvane, Serna, Dube, \& Stromer, 2000). A teoria aponta para a necessidade de se planejar procedimentos e arranjos experimentais para garantir que o comportamento dos sujeitos fique sob controle das mesmas propriedades da tarefa planejadas pelo experimentador para assumir controle.

Quando o controle de estímulos desenvolvido é incoerente com o planejamento experimental, resultados negativos podem ser obtidos em testes de desempenhos emergentes (por exemplo, testes de identidade generalizada ou de formação de classes), não em função de incapacidade do organismo testado, mas pelo fato de que o controle de estímulos supostamente em teste não foi desenvolvido ou está em conflito com outras relações de controle que podem prevalecer (McIlvane et al., 2000).

Os estudos que documentaram a interferência do controle pela posição dos estímulos no procedimento de emparelhamento ao modelo sugeriram a introdução de modificações de procedimento que visavam a prevenir essas incoerências de controle: (1) a introdução da variação da posição do estímulo modelo e (2) a ampliação da variação da posição dos estímulos de comparação (Galvão et al., 2002).

O presente estudo documenta a interferência do controle pela posição dos estímulos mesmo nessas condições de grande variação da posição dos estímulos modelo e comparação no treino. Descrevem-se manipulações experimentais realizadas na tentativa de identificar e contornar a interferência de controle pela posição dos estímulos em treino de emparelhamento arbitrário ao modelo com um macaco-prego como sujeito.

\section{Sujeito}

\section{MÉTODO}

Foi utilizado um macaco-prego (Sapajus sp.) chamado Louis, adulto, macho, com aproximadamente seis anos de idade no início do estudo.O sujeito apresentava história experimental em: 1) Mudanças repetidas de discriminações simples com três estímulos (Santos, 2003); 2) MTS por identidade e testes de identidade generalizada (Santos, 2003); 3) MTS arbitrário com duas escolhas utilizando o procedimento de modelagem do estímulo modelo (c.f. Zigmont, Lazar, Dube, \& McIlvane, 1992) e teste de simetria (Kataoka, 2005; Santos, 2003); 4) MTS arbitrário (Kataoka, 2005) com o procedimento de máscaras (c.f. McIlvane, Kledaras, Munson, King, De Rose, \& Stoddard, 1987).

O sujeito ficava alojado com outros dois indivíduos da mesma espécie em uma gaiola-viveiro, no biotério pertencente à Escola Experimental de Primatas da Universidade Federal do Pará. $\mathrm{O}$ animal tinha livre acesso à água, recebia refeição duas vezes ao dia. Os procedimentos 
de manejo e manutenção do animal foram aprovados pelo Comitê de Ética em Pesquisa com Animais do Centro de Ciências Biológicas da Universidade Federal do Pará.

\section{Equipamento e Ambiente Experimental}

Foi utilizada uma câmara experimental medindo $0,80 \times 0,80 \times 0,70 \mathrm{~m}$. O assoalho, o teto e a parede lateral esquerda da câmara experimental eram construídos de tela de aço tipo moeda. A parede lateral esquerda tinha uma porta de $0,35 \times 0,20 \mathrm{~m}$, que funcionava como entrada e saída do sujeito. Na parede frontal da câmara experimental, localizava-se uma abertura de $0,26 \times 0,26 \mathrm{~m}$, na qual foi acoplado um monitor com tela sensível ao toque que possibilitava a apresentação dos estímulos de acordo com as condições experimentais.

Um dispensador automático de pelotas de $190 \mathrm{mg}$ foi utilizado para consequenciar as respostas corretas. Através de uma mangueira, a pelota chegava até uma bandeja situada $24 \mathrm{~cm}$ abaixo da tela do computador. Logo acima da bandeja do comedouro, havia uma luz vermelha que acendia no momento em que o comedouro era acionado. A sala experimental era iluminada por uma lâmpada fluorescente. Além disso, a câmara tinha outra lâmpada fluorescente colocada do lado de fora do teto da gaiola experimental e que permanecia ligada durante a sessão.

\section{Estímulos}

Foram utilizados cinco estímulos visuais constituídos de formas desenhadas em linhas pretas sobrepostas em fundo cinza, assim nomeados: A1, A2, B1, B2 e MK (ver Figura 1) estímulo MK era um quadrado branco já apresentado em experimento anterior com a função de "máscara" (ou seja, comparação vazio, c.f. McIlvane et al., 1987). Todos os estímulos possuíam 3,8 $\mathrm{cm}^{2}$. O sujeito já possuía experiência anterior com os estímulos dos conjuntos A e B, num experimento que encontrou dificuldades de treino de emparelhamento arbitrário com o mesmo sujeito, em função de persistência de erros mesmo após muitas sessões de treino. Esse desempenho estável e impreciso impediu que os objetivos do experimento anterior fossem alcançados e motivou o presente experimento.

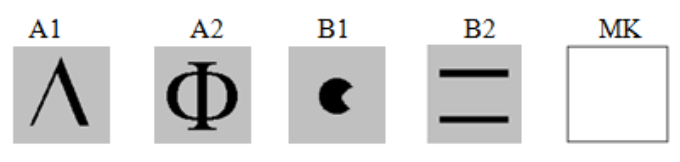

Figura 1. Estímulos discriminativos.

\section{Procedimento}

Treino de emparelhamento ao modelo arbitrário AlB1 e $A 2 B 2$.

Cada tentativa se iniciava com a apresentação do estímulo modelo (A1 ou A2) em qualquer uma das 9 posições (janelas) de uma matriz $3 \times 3$ na tela do monitor. Uma resposta de toque ao modelo produzia a remoção desse estímulo e a imediata apresentação de três estímulos de comparação em quaisquer das nove posições da matriz. Respostas ao estímulo definido pelo experimentador como $\mathrm{S}+$ produziam o acionamento do comedouro (com a apresentação de uma pelota de comida sabor banana) e o IET (6 segundos); respostas ao estímulo definido com Sproduziam o IET sem a apresentação de comida. As relações condicionais A1B1 e A2B2 foram reforçadas da seguinte forma:

(1) Dado o modelo A1, as seguintes combinações de S+ e S- eram apresentadas: B1+/B2-; B1+/MK-; $\mathrm{MK}+/ \mathrm{B} 2-; \mathrm{B} 1+/ \mathrm{B} 2-$ e $\mathrm{MK}-$; $\mathrm{MK}+, \mathrm{B} 2-$ e B2-.

(2) Dado o modelo A2, as seguintes combinações de S+ e S- eram apresentadas: B2+/B1-; B2+/MK-; MK+/B1-; B2+/B1- e MK-; MK+/B1- e B1-.

A introdução de comparações vazias (MK) no treino das discriminações condicionais fazia parte do procedimento do estudo anterior (Kataoka, 2005) e tinha como objetivo induzir o estabelecimento de relações de controle por S+e por S- no treino discriminativo (c.f. Goulart et al., 2005) e ainda funcionar como um passo intermediário para a introdução do procedimento de tripla escolha sem aumentar o número de relações treinadas, considerando que o sujeito tinha longa história de emparelhamento ao modelo com dupla escolha.

Tanto no estudo de Kataoka (2005), quanto no presente estudo, o número de vezes que cada estímulo foi apresentado nas 9 janelas, bem como o número de vezes que o S+ foi programado em cada janela não foi precisamente controlado. Isso possivelmente contribuiu para os resultados negativos nos testes de simetria no estudo de Kataoka (2005) e reproduziu aqui a persistência de alguns poucos erros a cada sessão conforme relatado no estudo anterior (ver seção de resultados). A obtenção de desempenho estável com alguns poucos erros sistematicamente verificados em diversas sessões de treino sugere a ocorrência de topografias de controle não planejadas pelo experimentador. $\mathrm{O}$ presente estudo apresenta manipulações com o objetivo de documentar possível interferência de controle pela posição dos estímulos, resultante da falha de controle experimental acima mencionada no estudo de Kataoka (2005).

Durante o estudo de Kataoka (2005) foram observados erros sistemáticos nas discriminações principalmente quando os estímulos $\mathrm{S}+$ eram apresentados nas posições 8 e 9 da matriz. Esses dados apontaram a possibilidade de interferência por controle não programado por posições (para detalhes, ver seção de resultados abaixo).

\section{Manipulações para Verificação de Relações de Controle}

Foram introduzidas manipulações da posição dos estímulos para avaliar se as posições em que os estímulos eram apresentados interferiam nos erros observados nas sessões, o que indicaria controle de estímulos incoerente com o planejado. Foram realizadas três etapas. Cada etapa consistiu de uma sessão com 60 tentativas de 
emparelhamento arbitrário com três comparações com reforço contínuo.

Manipulação 1: remoção de tentativas com $\mathrm{S}+$ na posição 8 das janelas.

As quatro tentativas em que o $\mathrm{S}+$ ocupava a posição 8 foram substituídas por tentativas em que o $\mathrm{S}+$ ocupava uma das posições de 1 a 7 . As demais tentativas da sessão permaneceram inalteradas. Essa manipulação teve o objetivo de avaliar se a mudança na posição do $\mathrm{S}+$ por si só produziria desempenhos corretos.

Manipulação 2: remoção de tentativas com $\mathrm{S}+$ nas posição 8 e 9 das janelas.

Adicionalmente ao que foi descrito na Manipulação 1, a tentativa em que o $\mathrm{S}+$ ocupava a posição 9 foi substituída por outra tentativa em que o $\mathrm{S}+$ ocupava uma das posições de 1 a 7 . O objetivo foi o mesmo apresentado no teste anterior.

Manipulação 3: retorno de tentativas com $\mathrm{S}+$ nas posições 8 e 9 das janelas.

Depois de verificar se o desempenho do sujeito mudaria nas Manipulações 1 e 2, a Manipulação 3 teve como propósito avaliar se o retorno ao contexto de treino anterior (no qual havia tentativas nas quais o $\mathrm{S}+$ aparecia nas posições 8 e 9 e os $S$ - nas posições que iam de 1 a 7) produziria desempenhos incorretos iguais aos obtidos naquele treino.

\section{RESULTADOS}

Treino de emparelhamento ao modelo arbitrário AlB1 e A2B2.

A Figura 1 mostra a precisão média do desempenho do sujeito nas últimas três sessões do treino de relações condicionais arbitrárias com $\mathrm{S}+$ apresentado nas posições 8 e 9 e a porcentagem de acertos em cada uma das manipulações realizadas.

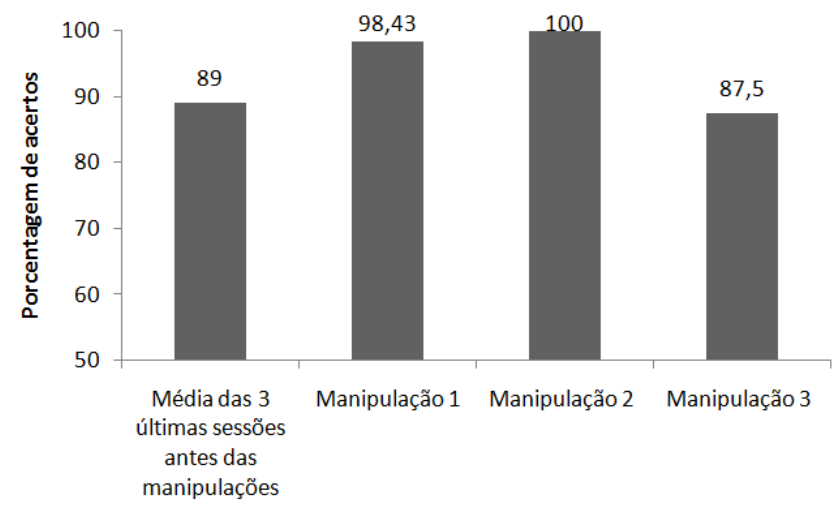

Figura 2. Porcentagem de acertos nas discriminações arbitrárias sob treino e avaliação. A primeira barra mostra a média de acertos nas três sessões experimentais que precederam as manipulações. As demais barras mostram a precisão do desempenho em cada uma das manipulações feitas.
Análise minuciosa dos erros mostrou que, nas tentativas em que o $\mathrm{S}+$ era $\mathrm{B} 1$ ou B2 apresentado nas posições 8 ou 9 da matriz 3 x 3, sendo os S- (B1 [ou B2] e MK) apresentados em duas posições entre 1 e 7, o sujeito errava sistematicamente, escolhendo sempre o estímulo MK. Das 60 tentativas que compunham cada sessão, cinco apresentavam a configuração descrita anteriormente (quatro tentativas com o $\mathrm{S}+$ na posição 8 e uma tentativa com o S+ na posição 9). Especificamente nestas tentativas, a precisão do desempenho foi nula durante seis sessões consecutivas. Neste tipo de tentativa, as respostas sempre foram na MK. Nas demais tentativas, inclusive nas que o $\mathrm{S}+$ aparecia nas posições 8 ou 9 , mas os $\mathrm{S}$ - eram dois estímulos iguais (por exemplo: B1+/B2- e B2-) a precisão do desempenho foi sempre de $100 \%$ de acertos.

\section{Manipulações para Verificação de Relações de Controle}

$\mathrm{Na}$ Manipulação 1, sem apresentação de $\mathrm{S}+$ na Posição 8 , a precisão do desempenho foi de $98,43 \%$ de acerto, ou seja, apenas um erro foi registrado no total de 60 tentativas. Esse erro ocorreu na tentativa em que o $\mathrm{S}+$ foi apresentado na posição 9, e os $S$ - nas posições de 1 a 7 .

$\mathrm{Na}$ Manipulação 2, sem apresentação de $\mathrm{S}+$ nas Posições 8 e 9, o sujeito apresentou $100 \%$ de acerto nas 60 tentativas da sessão.

$\mathrm{Na}$ Manipulação 3, na qual houve o retorno de tentativas com $\mathrm{S}+$ nas posições 8 e 9 , o sujeito apresentou escolhas incorretas em todas as tentativascom $\mathrm{S}+$ nas posições 8 e 9, mantendo $100 \%$ de acertos nas demais tentativas. $\mathrm{O}$ estímulo $\mathrm{MK}$ foi selecionado em todas as tentativas que continham como comparações os estímulos B1, B2 e MK quando o $\mathrm{S}+(\mathrm{B} 1$ ou B2) era apresentado nas posições 8 e 9 da matriz. Assim, foi obtido o mesmo percentual de acerto das sessões que precederam as manipulações.

\section{DISCUSSÃO}

Os dados aqui apresentados mostram que, mesmo com variações de posições do estímulo modelo e comparações em nove posições de uma matriz 3x3, é possível encontrar interferência do controle pela posição dos estímulos na precisão do desempenho.

É possível que o número de vezes que o $\mathrm{S}+$ foi apresentado nas posições 8 e 9 no estudo de Kataoka (2005) tenha sido baixo se comparado ao número de vezes que foi apresentado nas demais posições. Embora o levantamento preciso das posições em que os estímulos foram apresentados em cada uma das tentativas de todas as sessões prévias de treino não tenha sido possível, essa falha no balanceamento foi confirmada por Kataoka (2005) com respeito à posição 9 em uma pequena amostra de sessões no final do treino. Esse desbalenceamento poderia ter controlado o repertório de observação do sujeito (scan) aos estímulos ao longo do display. O scan só abrangia toda a 
tela (incluindo as posições 8 e 9) quando o S+ não era encontrado nas demais posições.

Sustentando essas interpretações, está o fato de que nem sempre o sujeito apresentava escolhas incorretas quando a comparação positiva era apresentada na linha inferior da tela do computador. Em tentativas com configuração desconhecida (um estímulo $\mathrm{S}+\mathrm{e}$ dois estímulos S- iguais) para o sujeito, mesmo quando o $\mathrm{S}+$ aparecia na porção inferior, a resposta de "escaneamento" emitida era suficiente para permitir escolhas corretas. Por exemplo, tendo A1 como modelo e B1 como S+ apresentado juntamente com dois exemplares de B2 como S- em diferentes posições da matriz $3 \times 3$, as escolhas eram sistematicamente corretas. Quando o sujeito encontrava dois exemplares de $+-2(\mathrm{~S}-)$, continuava a "escanear" a tela, escolhendo o $\mathrm{S}+$ mesmo nas posições 8 e 9 .

É provável que o estabelecimento de relações de controle de estímulo incoerentes com o planejamento experimental, como relatado aqui, explique boa parte dos resultados inconsistentes já obtidos em trabalhos buscando a demonstração de identidade generalizada (Iversen, 1997) e a formação de classes de equivalência em não-humanos e humanos com desenvolvimento severamente atrasado (Devany, Hayes, \& Nelson, 1986; Lionello \& Urcuioli, 1998; Lionello-DeNolf \& Urcuioli, 2002; Sidman \& cols., 1982).

O conceito de coerência de controle de estímulos ajuda a compreender características sutis do repertório discriminativo do organismo, assim como o fracasso frequente ao se tentar obter, por exemplo, altos níveis de desempenho em macacos-prego expostos ao treino de discriminações condicionais arbitrárias usando-se o procedimento padrão de matching-to-sample (MTS) com atraso zero (Barros \& Galvão, 2002; Barros, Galvão, Brino, Goulart, \& McIlvane, 2005).

O presente estudo é um exemplo da possibilidade de se criar procedimentos de teste eficazes para verificar as relações de controle de estímulos estabelecidas durante um treino, envolvendo avaliação minuciosa e frequente dos resultados das sessões experimentais, visando a reestruturação do procedimento de treino aplicado para cada sujeito (Galvão, Barros, Goulart, Mendonça, \& Rocha, 2002; Sidman, 1988/1960). A aplicação do procedimento de avaliação aqui descrito permitiu que se atribuíssem os erros do sujeito no re-treino de linha de base à rejeição do S-, em conformidade com sua história de treino prévia envolvendo duas escolhas, erros que de outra forma poderiam ter sido incorretamente atribuídos ao desenvolvimento de um controle pela máscara.

A utilização do procedimento de máscara para estabelecer relações de controle coerentes com as planejadas pelo experimentador (neste caso, controle misto), não impediu que outras relações concorrentes de controle de estímulos se estabelecessem e fossem mantidas. A análise dos resultados ao longo do treino evidenciou as peculiaridades do comportamento do sujeito que levaram a decisões de alterar o procedimento para eliminar as incoerências entre as relações de controle de estímulos planejadas pelos experimentadores e aquelas que efetivamente ganhavam controle sobre o responder do sujeito e garantir a aprendizagem efetiva do repertório que se buscava ensinar. A efetividade desse conjunto de procedimentos como uma tecnologia de ensino de comportamentos complexos a sujeitos não-humanos e humanos com desenvolvimento atípico caracteriza um exemplo de engenharia comportamental com possibilidades de ser traduzido para pesquisas com humanos com dificuldades de aprendizagem.

\section{REFERÊNCIAS}

Barros, R. S., \& Galvão, O. F. (2002). Estratégias para identificação de características sutis do repertório discriminativo em macacos-prego (Cebus apella). Em: Anais do XX Encontro Anual de Etologia, Natal-RN, 2002, 64-68.

Barros, R. S., Galvão, O. F., Brino, A. F., Goulart, P. R. K., \& Mcllvane, W. J. (2005). Variáveis de procedimento na pesquisa sobre classes de equivalência: contribuições para o estudo do comportamento simbólico. Revista Brasileira de Análise do Comportamento, 1, 15-27.

Devany, J. M., Hayes, S. C., \& Nelson, R. O. (1986). Equivalence class formation in language-able and language-disabled children. Journal of the Experimental Analysis of Behavior, 46, 243-257.

Dube, W. V., \& McIlvane, W. J. (1996). Some implications of a stimulus control topography analysis for emergent stimulus classes. In T. R. Zental \& P. M. Smeets (Eds.), Stimulus class formation in humans and animals (pp. 197-218). North Holland: Elsevier.

Galvão, O. F., Barros, R. S., Goulart, P. R. K., Mendonça, M. B., \& Rocha, A. C. (2002). Escola experimental de primatas. Estudos de Psicologia (Natal), 7, 361-370.

Goulart, P. R. K., Mendonça, M. B., Barros, R. S., Galvão, O. F., \& McIlvane, W. J. (2005). A note on select- and reject-controlling relations in the simple discrimination of capuchin monkeys (Cebus apella). Behavioural Processes, 69, 295-302.

Iversen, I. H. (1997). Matching-to-sample performance in rats: a case of mistaken identity? Journal of the Experimental Analysis of Behavior, 68, 27-45

Iversen, I. H., Sidman, M., \& Carrigan, P. (1986). Stimulus definition in conditional discriminations. Journal of the Experimental Analysis of Behavior, 45, 297-304.

Kataoka, K. B. (2005). Relações de controle por seleção em relações condicionais arbitrárias e a obtenção de relações emergentes com macaco-prego (Cebus apella). Relatório Técnico Científico. Programa Institucional de 
Bolsas de Iniciação Científica - PIBIC. Universidade Federal do Pará.

Lionello, K. M., \& Urcuioli, P. J. (1998). Control by sample location in pigeons matching to sample. Journal of the Experimental Analysis of Behavior, 70, 235-251.

Lionello-DeNolf, K. M., \& Urcuioli, P. J. (2002). Stimulus control topographies and tests of symmetry in pigeons. Journal of the Experimental Analysis of Behavior, 78, 467-495.

Mcllvane, W. J., \& Dube, W. V. (2003). Stimulus control topography coherence theory: Foundations and extensions. The Behavior Analyst, 26, 195-213

McIlvane, W. J., Kledaras, J. B., Munson, L, C., King, K, A., de Rose, J. C., \& Stoddard, L. T. (1987). Controlling relations in conditional discrimination and matching by exclusion. Journal of the Experimental Analysis of Behavior, 48, 187-208.

McIlvane, W. J., Serna, R., Dube, W. V., \& Stromer, R. (2000). Stimulus control topography coherence and stimulus equivalence: reconciling test outcomes with theory. In J. Leslie \& D. E. Blackman (Eds), Issues in experimental and applied analysis of human Behavior. Reno: Context Press.

Reynolds, G. S. (1961). Attention in the pigeon. Journal of the Experimental Analysis of Behavior, 4, 57-71.

Santos, J. R. (2003). Cognição Animal: Identidade Generalizada e Simetria em macaco-prego. Dissertação de Mestrado, Belém: Universidade Federal do Pará.

Sidman, M. (1988). Tactics of scientific research: Evaluating experimental data in Psychology. Boston, MA: Authors Cooperative. (Trabalho originalmente publicado em 1960).

Sidman, M. (1992). Adventitious control by the location of comparison stimuli in conditional discriminations. Journal of the Experimental Analysis of Behavior, 58, 173-182.

Sidman, M., Rauzin, R. M., Lazar, R., Cunninghan, S., Tailby, W., \& Carrigan, P. (1982). A search for symmetry in the conditional discriminations of rhesus monkeys, baboons, and children. Journal of the Experimental Analysis of Behavior, 37, 23-44.

Zigmont, D. M., Lazar, R. M., Dube, W. V., \& McIlvane, W. J. (1992). Teaching arbitrary matching via sample stimulus-control shaping to young children and mentally retarded individuals: A methodological note. Journal of the Experimental Analysis of Behavior, 57, 109-117.

Submetido em 26/09/2012. Aceito em 03/08/2013. Publicado em 01/02/2016. 